S. M. Zlepko, Doctor of Science (Eng.), Professor; S. V. Tymchyk, Cand. Sc. (Eng.), Assistant Professor; M. I. Palamarchuk; L. G. Koval, Cand. Sc. (Eng.), Assistant Professor; A. L. Dushkevych

\title{
3D-BIOPRINTING AND DEVELOPMENT OF THE DENTAL IMPLANTATION CLASSIFICATIONS
}

Regenerative medicine as an independent branch was formed at the end of the last century. The given scientific direction comprises: cellular technologies, cellular therapy, sub-cellular surgery, targeted therapy, 3Dbioprinting, dental implantology, etc.

Discoveries in the sphere of medical-biological science during the recent thirty years and modern information technologies promoted the development of the regenerative medicine, which occupies leading positions in science. From the point of view of organization-systemic positions the regenerative medicine can be considered as a complex system of scientific-engineering, practical and bioethical measures, which, using the mechanisms of the tissue restoration, regeneration, morphogenesis, available in human organism, is able to regenerate adequately the structures and functions of the injured organ or tissue.

Proteomic studies, research, using cellular technologies, tissue engineering, technology of creating biomaterials, 3D-printing and other modern technologies are referred to the most advanced directions of the regenerative medicine development. However, the availability of numerous classifications creates the information contradiction, when, on the one hand, the more classifications are used, the more information the doctor gets, and on the other hand - the more separate classifications are proposed to the doctor, the greater is the probability of taking the multivalued decision as a result of the redundant information, which will be different for each specialist, both by the volume and by the content.

The research contains the integrated classification of the means of dental implantation, based on new class of the dental implants-intelligent dental implants and three new classification features, that provided the solution of the information contraction and achieving the information balance, elimination of the multivalued decisions and the redundant information.

Key words: bioprinting, 3D-dental implantation,implants, regenerative medicine.

\section{Introduction}

Discoveries, made in modern medical-biological science during the last thirty years and rapid development of the information technologies promoted rapid progress of the regenerative medicine, that occupies leading positions in science. From the point of view of the organization-systematic approach the regenerative medicine can be considered as the complex system of the scientific-engineering, practical and bioethical measures, which, using the mechanisms of tissue restoration, regeneration, morphogenesis, available in human organism, is able to regenerate adequately the structures and functions of the injured organ or tissue [1].

Proteomic studies, research, using cellular technologies, tissue engineering, technology of biomaterials creation, 3D-printing and other modern technologies, which only yesterday were considered as unrealizable, are referred to the most advanced directions of the regenerative medicine development [1].

\section{Analysis of the literature sources}

Regenerative medicine, as the separate independent scientific direction was formed at the end of the last century, when the American biologist William Haseltine suggested using the term «regenerative medicine» as the basic component of new promising direction in the sphere of biomedical research [1]. The direction comprises: cellular technologies and cellular therapy [11]; subcellular surgery [1]; targeted therapy [12]; 3D-bioprinting (formed only in 2006 due to the development by the company Organovo (USA) of the first 3D-bioprinter) [1]; dental implantology, etc.

With every passing year doctors are more and more interested in 3D-bioprinting, its technology consists of the same elements as the classic printing, the analogy of the text in it is the digital model of the human organ, ink - cellular spheroids, paper - hydrogels, hand press 3D-bioprinter, which by its 
essence is the robot [9].

Actually jet printing can be considered as the step to the possibility of printing the human cells, based on the concept that became the basis of the jet printing and was discovered as far back as in 1833 by Felix Savart [9].

In 1985 Mykhailo Feigin suggested the principle of the layer-by-layer formation of the volume modules by means of baking of such materials as: film, polyester, plastic materials, paper, composites. Serious drawback of the suggested method was the roughness of the external surface and problems of the excessive materials elimination [9].

In 1986 Charles Hull patented installation that used stereo lithography (SLA) and the base that is photo polymerized. The installation was commercialized in 1987. Although STL-installations are used nowadays, high cost slows down their wide application [9].

In 1987 Israel company Cubitall continued working with layers, formed by means of baking from the powder with the help of the selective laser action. The idea consists in layer-by- layer application of the photopolymer material on the template, the photopolymer solidified under the impact of the ultraviolet. The cavities of the object were filled with the wax and in such a manner the whole object was regenerated in the volume [9].

And, at last, in 1988, Scott Crump patented the technology of layer-by-layer filling of the extruded melt (FDM), according to this technology, the melt plastics, metal, wax is supplied across the extruder in the form of the string, which is put layer- by- layer on the working table, forming the object [9].

For the bioprinting the classification of the types of printing, consisting of the jet, micro-extruding and laser-based printing is typical. Jet printing technologically does not differ from classic one and jet technology provides that the dispersed material - it is a liquid. At the same time, the result of the bioprinting is the stable 3D structure with the sufficient density of cells, that requires the additional stage of the structure crosslinking, that increase the time and reduces the rate of printing [9].

Jet printing, as the bioprinting technology, is optimal for the regeneration of the skin surfaces and cartilages and high rate of printing enables to print the cells directly on the damaged section.

Microextruding printing is the technology, based on the distribution of the bubbles of the substance along the axes $\mathrm{x}, \mathrm{y}, \mathrm{z}$, it originates from the extruding technology of 3D printers and enables to achieve in bioprinting the required physiological density of the cells, needed for the creation of the three dimensional objects [9].

Laser printing is the technology, built on the principles of the direct laser- inducted transfer. Principle of LAB (Laser-Assisted bioprinting) devices operation consists in the transfer from the substrate with laser-absorbing layer on the substrate of the collector of the cellular material by means of the focused laser beams [9].

Classification of the types of implantation, based on such classification features as : according to the type of the interaction of the implant with soft and hard tissues of the organism [2]; according to the time of the implant placement after the extraction of the tooth or root [3]; according to the character of the interaction of the central implant with mouth cavity [3] is most widely used in dental implantology.

We will consider the given classification in details.

I. According to the type of the interaction of the implant with soft and hard tissues of the organism [2].

1. Endodonto - endosteal implantation: the implant is the pin with different elements for its fixation. For the first time it was suggested in 1943.

2. Endosteal implantation: the implant has the form of the helix, cylinder or plate and is used on both jaws. The technique was developed in 1967.

3. Subperiostal implantation: first the cast is taken from the bone, then individual implant is fabricated, which is placed under the supra bony flap. For the first time it was described in 1949.

4. Intramucosal or insert implantation: metal implant in the form of the button is introduced in the mucous coat on the alveolar bones for the fixation of the complete denture. Such implantation was performed for the first time in 1940. 
5. Submucous or mucous membrane implantation: introduction of the magnets into the transitory fold for the retention of the denture. Such kind of the implantation was developed by Popov in 1973.

II. According to the time of the implant placement after tooth or root extraction [3].

1. Immediate implantation, the implant is introduced into the alveolar socket immediately after its extraction.

2. Delayed implantation, the implant is placed into the bone site of the intact osseous tissue, which is formed by the doctor.

Ill. According to the feature of the interaction of the central implant with the mouth cavity [3].

1. Single stage or single component implantation.

2. Two -stage implantation, at the first stage the body of the implant is placed, after implant "survival", its head is installed.

In practical dental implantation screw and cylindrical implants got wide application [2], these implants may be demountable and integral. They are represented by the qualification, where the qualification feature is the form of the implant and state of osseous tissue [3, 7].

1. Endosseous implants in the form of the root of the natural tooth are used when the thickness of the osseous tissue is normal.

2. Endosseous implants, which have the form of thee plate (leaf-tailed) are used if the osseous tissue is thinned.

3. Endosseous combined type of the implants consists the implants which have the form of the root and plate.

4. Subperiostomal implants are introduced between the bone and periosteum.

5. Implants, placed in the mucous coat, are used, generally, for the fixation of the already installed dentures.

6. Cylindrical implants, complete or hollow.

7. Screw - implants and conical implants.

Classification of the dental implants: a) according the material, they are made of $[3,7]$.

1. Metal.

2. Ceramic.

3. Sitall.

4. Plastic (are not used in pure form).

5. Combined.

b) According the structure of the material:

1. Nonporous.

2. Surface porous.

3. Perforated.

4. Combined.

The existing problem is in the information contradiction when, on the one hand, the more qualification are used, the more information the doctor obtains, but on the other hand the more separate classifications are proposed to the doctor, the greater is the probability of making multivalued decision as a result of redundant information, which will be different for each doctor both by the volume and the content.

Aim of the paper - create integrated classification of the means of dental implantation, based on new class of the dental implants - intelligent dental implants.

\section{Main part}

As it was mentioned in [10] great loads on the implants, negative impact of the unforeseen external and internal factors, violation of technologies of the dental implantation at the phase of osteointegration, errors at the stage of the abutment manufacture cause mechanical damage of the implant state directly in the gingival tissue, emergence of the inflammatory process and other undesirable complications. It is possible 
to avoid the above-mentioned [10], at least, by means of periodical or continuous control of the implant state, rate of the reparative regeneration of the injured bone tissue, level of the implant biocompatibility during its life cycle, etc. All this can be achieved by means of introduction of the biological feedback across the radio channel, without causing the patient the discomfort and is the basis of a new functional class of the dental implants - intelligent implants. Basic components of the intelligent implants are intelligent biosensors, biotelemetric (radiofrequency) transmitting/receiving modules and power supply elements, which are implanted.

The introduction of new classification feature of the dental implantation - by the function of the implant state control and, as a result, the emergence of two new characteristics of the implants: by the type of the installed implant state control and the technology of 3D-printing stipulated the necessity to improve the existing classifications of the dental implantation by means of their integration into a single integrated classification, its structure is shown in Fig. 1.

The suggested classification is flexible and open both for further development and expansion and for any manipulations inside it. The introduction of the classification feature by the types of the installed implant state control stipulated the necessity of implementation of the additional control modes: periodic examinations (performed by the doctor, using minimal amount of the tools periodically or, if necessary ,when the patient applies); periodical instrumental (performed by the doctor, using the necessary electronic, mechanical or combined tooling periodically or if necessary (when the patient applies); continuous monitoring - performed in automatic mode, using radio channel and biological feedback and provides objective fixation of the mechanical breaking of the implant or the start of the inflammatory process, providing early determination of the pathology and its treatment.

As a rule, the implant placement operations provide the obligatory preparation stage, at this stage the patient passes the necessary diagnostics and, if necessary, stomatologic and orthodontic treatment. At the same time, within the limits of the given stage the correct structure of the jaw is regenerated, the conclusions regarding the state of gingivae and the level of their readiness for the implantation is made by the parodontist, possible risks, indications and contra-indication are assessed and considered [5].

Thus, on the condition of the observing the above-mentioned measures and application of the integrated classification the basic algorithm of the dental implantation [5] has the following form:

a) preparative-diagnostic stage;

b) regeneration stage - realization of the regeneration procedures and preparation of the mouth cavity for the implant placement;

c) new stage: stage of 3D-modeling and bioprinting of the jaw with the implant;

d) surgical stage - implant placement on the condition of the obligatory availability of the sufficient amount of the osseous tissue;

e) implant survival stage- control over the renovation of the blood microcirculation in the tissues of gingiva and jaw, assessment of the implant state, formation of the forecast of the implant complete survival;

f) stage of dental prosthetics - mounting of the abutment and crown;

g) new stage:stage of the long-term monitoring and forecast of the implant and jaw state. 

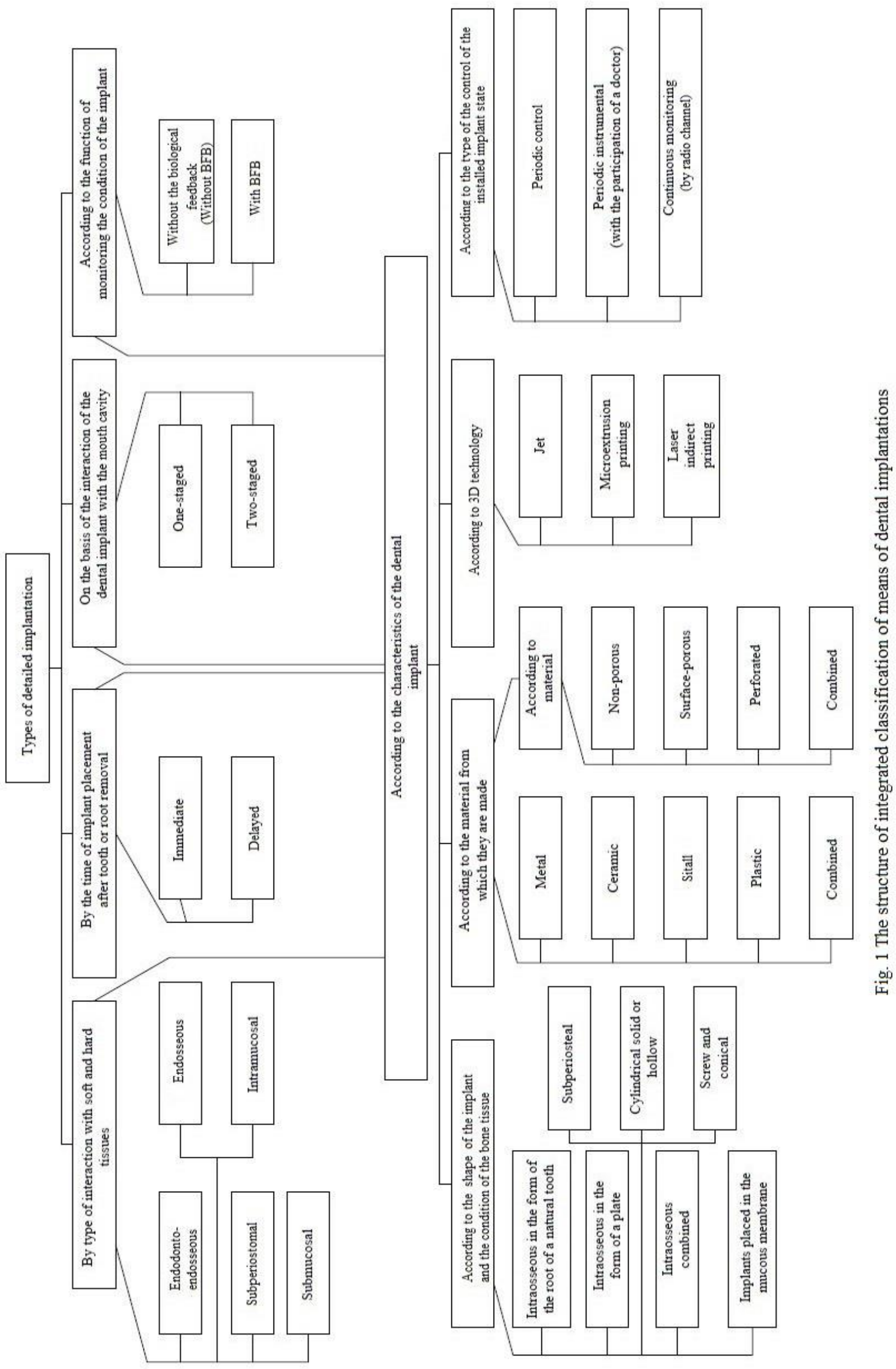

Scientific Works of VNTU, 2020, № 3 


\section{Conclusions}

Creation of the integrated classification of the dental implantation means on the base of a new class of the dental implants - intelligent dental implants and three new classification features: by the function of the implant state control, by the type of the installed implant state control and by the technology of 3D-printing provided the solution of the information contradiction and achieving the information balance, elimination of the multivalued decisions and redundant information.

\section{REFERENCES}

1. Modern directions and prospects of the regeneration medicine development [Electronic resource] / E. A. Pronina, E. B. Popykhova, T. V. Stepanova, A. N. Ivanov // Modern problems of science and education. - 2019. - №3. - Access mode : http://www.science-education.ru/ru/article/view?id=28968. (Rus).

2. Theoretical aspects of the dental implantation [Electronic resource] / Access mode : https://knowledge.allbest.ru/medicine/2c0a65625b3bd68b5c53a88521216d36_0.html. (Rus).

3. Dental implants [Electronic resource] / Access mode : https://dentekua.com/product-category/mis-uk/hirurgiyauk/?gclid=CjwKCAiAzNj9BRBDEiwAPsL0d_I-ZZsz34zQDEynbigBfS3WJ8egVkhTrVZ3W0Q11KswAOhrv1BgRoCMOMQAvD_BwE. (Rus).

4. Popkov A. V. Biocompatible implants in the traumatology and orthopedics (literature review) / A. V. Popkov. - Genius of the orthopedics. - 2014. - №3. - P. 94 - 99. (Rus).

5. Treatment by Non-Grumbling Dental Drilling Machine( about the implantation) [Electronic resource] / V. Avetov // Gazeta.ru. - Access mode : https://www.gazeta.ru/science/2010/12/10_a_3461173.shtml. (Rus).

6. Minina A. N. Fundamentals of the dental implantation : Study-guide / A. N. Minina, T. N. Chernina. - Vitebsk : VSMU, 2013. - 76 p. (Rus).

7. Dental implants: biomechanical aspect of the development / S. Zlepko, D. Shtofel, S. Tymchyk, I. Lula // Prospects of the machine-building and transport development : International scientific- engineering conference, 2019 : Collection of the abstracts. - Vinnytsia : PE «TV Edelveise and K», 2019. - P. 134 - 136. (Ukr).

8. Printing of the future: bioprinting. Part 2. [Electronic resource] / Access mode : https://pechatnick.com/articles/pechatbydyshego-bioprinting-chast-2. (Rus).

9. Printing of the future: bioprinting. Part 1. [Electronic resource] / Access mode : https://pechatnick.com/articles/pechatbydyshego-bioprinting-chast-1. (Rus).

10. Intelligent implants in stomatology: realities and prospects / S. Zlepko, S. Tymchyk, O. Grushko, I. Vishtok // Prospects of machine-building and transport development : International scientific-engineering conference, 2019. : Collection of abstracts. - Vinnytsia : PE «TV Edelveise and K», 2019. - P. 131 - 132. (Ukr).

11. Yamanaka S. Nuclear reprogramming to a pluripotent state by three approaches / S. Yamanaka, H. M. Blau // Nature. 2010. - Vol. 465. - P. $704-712$.

12. Immunosuppressive action of mesenchymal stem (stromal) cells / I. V. Majanskaya, A. Ya. Goganova, N. I. Tolkacheva [at all] // Immunology. - 2013. - V. 34, №2, P. 122 - 128. (Rus).

Editorial office received the paper 12.03.2020.

The paper was reviewed 19.03.2020.

Zleplko Sergey - Doctor of Science (Eng.), Professor, Head of the Chair of Biomedical Engineering.

Tymchyk Sergey - Cand. Sc. (Eng.), Assistant -Professor with the Department of Biomedical Engineering.

Palamarchuk Mykhaylo - Post Graduate with the Department of Biomedical Engineering.

Koval Leonid - Cand. Sc. (Eng.), Assistant -Professor with the Department of Biomedical Engineering. Vinnytsia National Technical university.

Dushkevich Andriy - Doctor of Dental Surgery. 